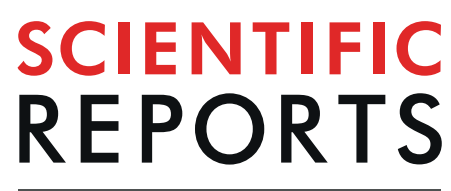

\title{
Using $\mathrm{SpO}_{2}$ Recovery Index after a 6-Minute Walk Test to Predict Respiratory-Related Events in Hospitalized Patients with Interstitial Pneumonia
}

\author{
Yasunari Sakai ${ }^{1 *}$, ShuheiYamamoto ${ }^{1}$, Miho Hoshina ${ }^{1}$, Shohei Kawachi ${ }^{1}$, Takashi Ichiyama $^{2}$ \& \\ Masayuki Hanaoka²
}

Although the prognostic factors of interstitial pneumonia (IP) patients have been reported, IP has poor prognosis. Hospitalized patients with IP have severely impaired pulmonary diffusion capacity and prominent desaturation. We hypothesized that determining oxygen saturation recovery $\left(\mathrm{SpO}_{2}\right.$ recovery index) after the 6-minute walk test (6MWT) can provide additional prognostic information regarding rehospitalization for respiratory-related events. We evaluated 73 IP patients at our hospital for demographic characteristics, pulmonary function tests and 6MWT. The Kaplan-Meier method was used to estimate rehospitalisation for respiratory-related events using $\mathrm{SpO}_{2}$ recovery index. Cox regression analysis revealed a relationship between $\mathrm{SpO}_{2}$ recovery index and rehospitalisation. The optimum cutoff value of $\mathrm{SpO}_{2}$ recovery index was $4 \%$ (sensitivity, $71.4 \%$; specificity, $79.2 \%$ ). $\mathrm{SpO}_{2}$ recovery index was most closely related to pulmonary diffusion capacity $(r=0.684, P<0.001)$. In a multivariable model, it was the strongest independent predictor of rehospitalisation for respiratoryrelated events (hazard ratio, $0.3 ; 95 \%$ confidence interval, $0.10-0.90 ; P=0.032$ ). In this study, we estimated pulmonary diffusion capacity using $\mathrm{SpO}_{2}$ recovery index values obtained from $6 \mathrm{MWT}$. A SpO recovery index of $<4 \%$ can be useful in predicting rehospitalisation for respiratory-related events.

Central pathophysiological features of interstitial pneumonia (IP) include impaired degradation of pulmonary diffusion capacity due to pulmonary interstitium and capillary bed disorders, ventilation perfusion ratio mismatch, and worsening gas exchange with exercise ${ }^{1}$. Dyspnea during exercise reduces exercise tolerance, ability to perform daily activities, and health-related quality of life ${ }^{2}$. Although prognostic factors for IP have been reported, IP has poor prognosis ${ }^{3-8}$, including reduced pulmonary diffusion capacity and hypoxemia are poor prognostic factors in patients with $\mathrm{IP}^{3,4}$. Also, measuring partial oxygen pressure $\left(\mathrm{PaO}_{2}\right)$ during exercise predicts survival in patients with $\mathrm{IP}^{5}$. Moreover, desaturation during the 6 -minute walk test (6MWT) is a prognostic factor for patients with IP in stable conditions ${ }^{6}$. Exercise-induced hypoxemia (EIH) of patients with IP is related to the pulmonary diffusion function, which is also one of the prognostic factors ${ }^{6-8}$.

A simple test to evaluate desaturation is used clinically in $6 \mathrm{MWT}^{9,10}$. Heart rate recovery (HRR) obtained from 6MWT has drawn attention as a prognostic factor in patients with IP and in those with chronic obstructive pulmonary disease and heart failure ${ }^{11,12}$. Heart rate recovery 1 (HRR1), obtained by subtracting heart rate (HR) after 1-min walk, from the rate at 6MWT end, showed the strongest significance for mortality ${ }^{13,14}$. Considering the physiologic response after exercise as a prognostic indicator is essential. Hospitalized patients with IP have severely impaired pulmonary diffusion capacity and prominent desaturation; changes in HR after exercise and desaturation evaluation are important. Studies on the relationship between mortality and clinical indicators during and after exercise included stable IP outpatients ${ }^{13,14}$. To our knowledge, no study has evaluated hospitalized IP-patient mortality. HR during and HRR after exercise and all-cause mortality are reported, but readmission/mortality predictive factors have not been considered, restricting acute IP exacerbation and 
respiratory failure ${ }^{15-17}$. During rehabilitation of hospitalised patients with IP with an unstable respiratory state, pulmonary diffusion capacity should be considered. Determining the breathing pattern, exercise oxygen saturation, post-exercise oxygen saturation recovery and HR changes is crucial because rehospitalisation for acute IP exacerbation and respiratory failure accounts for a majority of the deaths in this disease; therefore, the results of such evaluations constitute useful information for risk management. Therefore, we examined whether oxygen saturation after 6MWT in hospitalized patients with IP reflected pulmonary diffusion capacity. Oxygen saturation recovery after 6MWT was hypothesized to provide additional prognostic information regarding rehospitalization for respiratory-related events in these patients.

\section{Materials and Methods}

Ethical approval. The study protocol was approved by the ethics committee of Shinshu University (No. 3732) and conducted according to the Declaration of Helsinki (latest version). Written informed consent was obtained from all participants after detailed explanation of the study protocol.

Patient selection. Patients with IP who were hospitalized at Shinshu University Hospital in Japan during January 2015-July 2018 were selected. IP was diagnosed based on the American Thoracic Society/Infectious Diseases Society of America guidelines ${ }^{18}$.

Patients with underlying gait disturbance due to cerebral infarction/collagen vascular disease/occupational exposure/high-flow oxygen and those with recognized cognitive decline (23 points $>$ Mini-Mental State Examination $)^{19}$ were excluded.

Procedure. This study is a cohort study. Demographic characteristics (age, gender, body mass index; BMI), diagnosis (idiopayhic pulmonary fibrosis, acute interstitial pneumonia, combined pulmonary fibrosis and emphysema), treatment (prednisolone, supplemental $\mathrm{O}_{2}$ flow), pulmonary function tests, 6MWT and laboratory data on admission (krebs von den lungen-6, $\mathrm{PaO}_{2} / \mathrm{FIO}_{2}$ ratio) were collected upon hospital discharge. $6 \mathrm{MWT}$ was performed upon hospital discharge and indicators were collected. Pulmonary function tests, including spirometry and pulmonary diffusion capacity for carbon monoxide (DLco), and changes in nitrogen levels $\left(\Delta \mathrm{N}_{2}\right)$ were measured as previously described ${ }^{20}$. Forced vital capacity (FVC) and DLco were assessed according to the American Thoracic Society/European Respiratory Society (ATS/ERS) criteria; their results were reported as predicted value percentages ${ }^{21-23}$. Pulmonary function tests using CHESTAC-8900 (CHEST, Tokyo, Japan) were conducted. Pulmonary hypertension $(\mathrm{PH})$ was diagnosed based on the estimated pulmonary artery systolic pressure calculated using the simple Bernoulli equation [estimated pulmonary artery systolic pressure $=4 \times(3$ tricuspid regurgitation velocity $)^{2}+$ estimated right atrial pressure] with reference to trans tricuspid pressure gradient determined using echocardiography ${ }^{24}$.

6MWT. Walking path and criteria for terminating measurement was set by the ATS guidelines ${ }^{25}$. 6 MWT protocol was designed to accurately assess oxygen desaturation and to provide a clinically useful oxygen titration. All patients' baseline HR and saturation of percutaneous oxygen $\left(\mathrm{SpO}_{2}\right)$ using pulse oximetry (Pulsox-M, Teijin Ltd, Tokyo, Japan) were measured. For patient safety, the test was discontinued on noting unbearable chest pain/ shortness of breath/new arrhythmia development. To accurately assess $\mathrm{SpO}_{2}$, the respiratory therapist confirmed an acceptable pulse oximeter signal and pulsing of oximeter bar indicating synchrony between HR before beginning all tests. Patients walked on a level surface with gentle vocal encouragement using set phrases every 1 min. $\mathrm{SpO}_{2}$ and $\mathrm{HR}$ were continuously measured during 6MWT, and in all patients modified Borg scale at 6MWT end and distance were measured. Change in $\mathrm{SpO}_{2}\left(\Delta \mathrm{SpO}_{2}\right)$ was the difference between a subject's resting $\mathrm{SpO}_{2}$ and the lowest $\mathrm{SpO}_{2}$ during $6 \mathrm{MWT}$ (resting - lowest $\mathrm{SpO}_{2}$ ). $\mathrm{SpO}_{2}$ recovery index was the difference between a subject's $\mathrm{SpO}_{2}$ at $6 \mathrm{MWT}$ end $\left(<6 \mathrm{~min}\right.$ if the test was terminated for a low $\mathrm{SpO}_{2}$ ) and $1 \mathrm{~min}$ into recovery and dividing by the resting $\mathrm{SpO}_{2}:\left[\left(\mathrm{SpO}_{2} 1 \mathrm{~min}\right.\right.$ into recovery after $6 \mathrm{MWT}-$ lowest $\left.\mathrm{SpO}_{2}\right) /$ resting $\left.\mathrm{SpO}_{2}\right] \times 100$.

End point. Subjects were followed-up with after 12 months; any major respiratory-related events requiring hospitalization were recorded.

Statistical analysis. The subjects were classified into two groups: not-rehospitalization (event free) and rehospitalization due to respiratory-related events groups. Categorical and continuous data were compared using the $\chi^{2}$ and Mann-Whitney $U$ tests, respectively. Correlations between quantitative variables of the lowest $\mathrm{SpO}_{2}$, $\Delta \mathrm{SpO}_{2}, \mathrm{SpO}_{2}$ at $1 \mathrm{~min}$ after $6 \mathrm{MWT}$, distance, $\mathrm{SpO}_{2}$ recovery index, and \%DLco in the entire population were evaluated using the bivariate Pearson test. Rehospitalization $\mathrm{SpO}_{2}$ recovery index validity was determined considering rehospitalization and $\mathrm{SpO}_{2}$ recovery index as objective and explanatory variables, respectively. The receiver operating characteristic (ROC) curve and area under the ROC curve (AUC) were determined. An optimal cutoff point maximizing sensitivity and specificity for $\mathrm{SpO}_{2}$ recovery index was determined from sensitivity and specificity curve intersection ${ }^{26}$.

The product-limit method was used to derive rehospitalization for respiratory-related events; Kaplan-Meier curves were used to display rehospitalization for the study sample stratified by $\mathrm{SpO}_{2}$ recovery index. For multivariable analysis of rehospitalization due to respiratory-related events to develop the most parsimonious model, candidate variables included those with a $P<0.05$ on bivariate analysis. Cox regression analysis examined the relationship between $\mathrm{SpO}_{2}$ recovery index and rehospitalization; adjusting for demographic characteristics; $\mathrm{PH}$ diagnosis and complication; treatment; and physiologic, 6MWT, and laboratory data. Overfitting was avoided by reducing potential confounding factors including $\mathrm{SpO}_{2}$ recovery index to a single composite characteristic by applying a propensity score. 


\begin{tabular}{|c|c|c|c|}
\hline Variable & $\begin{array}{l}\text { Not readmission } \\
(\mathrm{n}=59)\end{array}$ & $\begin{array}{l}\text { Readmission } \\
(\mathrm{n}=14)\end{array}$ & $P$-value \\
\hline \multicolumn{4}{|l|}{ Demographics } \\
\hline Age (years) & $68.9(7.7)$ & $66.7(10.4)$ & 0.468 \\
\hline Men/women, n (\%) & $37(63) / 22(37)$ & $9(64) / 5(36)$ & 0.42 \\
\hline BMI $\left(\mathrm{kg} / \mathrm{m}^{2}\right)$ & $20.5(3.1)$ & $19.6(4.4)$ & 0.139 \\
\hline \multicolumn{4}{|l|}{ Diagnosis } \\
\hline IPF, n (\%) & $32(54)$ & $8(57)$ & 0.664 \\
\hline AIP, n (\%) & $19(32)$ & $4(29)$ & 0.657 \\
\hline CPFE, n (\%) & $8(14)$ & $2(14)$ & 0.382 \\
\hline Complication of PH, n (\%) & $12(20)$ & $4(28)$ & 0.036 \\
\hline \multicolumn{4}{|l|}{ Treatment } \\
\hline Use PSL (mg/day) & $29.7(17.4)$ & $30.7(11.9)$ & 0.993 \\
\hline $\mathrm{O}_{2}$ flow $(\mathrm{L} / \mathrm{min})$ & $1.6(1.3)$ & $2.8(1.5)$ & 0.016 \\
\hline \multicolumn{4}{|l|}{ Physiologic } \\
\hline FVC predicted (\%) & $68.0(19.2)$ & $48.1(10.6)$ & $P<0.001$ \\
\hline $\mathrm{FEV}_{1.0}(\%)$ & $75.3(14.8)$ & $69.6(14.7)$ & 0.17 \\
\hline DLco predicted (\%) & $46.5(14.8)$ & $32.6(16.5)$ & 0.006 \\
\hline$\Delta \mathrm{N}_{2}(\%)$ & $4.1(1.8)$ & $4.9(1.6)$ & 0.089 \\
\hline \multicolumn{4}{|l|}{ 6MWT data } \\
\hline Resting $\mathrm{SpO}_{2}(\%)$ & $95.0(1.6)$ & $94.1(1.9)$ & 0.187 \\
\hline Distance $(\mathrm{m})$ & $331.7(134.6)$ & $227.1(148.9)$ & 0.016 \\
\hline HRR1 (beat) & $18.6(8.0)$ & $13.9(5.6)$ & 0.026 \\
\hline Lowest $\mathrm{SpO}_{2}(\%)$ & $85.3(4.8)$ & $82.9(4.3)$ & 0.028 \\
\hline$\Delta \mathrm{SpO}_{2}(\%)$ & $9.5(4.9)$ & $11.2(3.8)$ & 0.072 \\
\hline $\mathrm{SpO}_{2} 1$ minute $(\%)$ & $90.1(5.0)$ & $86.6(4.6)$ & 0.011 \\
\hline $\mathrm{SpO}_{2}$ recovery index $(\%)$ & $5.1(1.8)$ & $3.8(1.8)$ & 0.026 \\
\hline Modifide Borg Scale & $3.5(1.5)$ & $4.6(2.2)$ & 0.065 \\
\hline \multicolumn{4}{|l|}{ Laboratory data } \\
\hline KL-6 (U/ml) & $1308(194.0)$ & 1528.5 (1017.0) & 0.674 \\
\hline $\mathrm{P} / \mathrm{F}$ ratio & $303.0(71.3)$ & $261.6(97.6)$ & 0.12 \\
\hline
\end{tabular}

Table 1. Clinical Characteristics. P-values for comparison between groups stratified on readmission. Data are counts (percentages), mean (SD). Definition of abbreviations: BMI indicates body mass index; IPF, Idiopayhic pulmonary fibrosis; AIP, Acute interstitial pneumonia; CPFE, Combined pulmonary fibrosis and emphysema; $\mathrm{PH}$, Pulmonary hypertension; PSL, Prednisolone; $\mathrm{O}_{2}$ flow, Supplemental $\mathrm{O}_{2}$ flow; HRR1, HR at the end of $6 \mathrm{MWT}$ minus $\mathrm{HR}$ after 1 minute at the end of $6 \mathrm{MWT} ; \Delta \mathrm{SpO}_{2}, \mathrm{SpO}_{2}$ at the end of the $6 \mathrm{MWT}$ minus $\mathrm{SpO}_{2}$ at baseline; $\mathrm{SpO}_{2} 1$ minute, $\mathrm{SpO}_{2} 1 \mathrm{~min}$ into recovery after $6 \mathrm{MWT} ; \mathrm{SpO}_{2}$ recovery index; $\left\{\left(\mathrm{SpO}_{2} 1\right.\right.$ minute in to recovery after 6MWT minus lowest $\mathrm{SpO}_{2}$ )/resting $\left.\mathrm{SpO}_{2}\right\} \times 100$; KL-6, Krebs von den Lungen-6; P/F ratio, $\mathrm{PaO}_{2} /$ $\mathrm{FIO}_{2}$ ratio.

Analyses were performed using SPSS 24.0 software (IBM Japan, Tokyo, Japan). Descriptive statistics [mean \pm standard deviation (SD)] were used. All tests assumed unequal variances; $P<0.05$ indicated statistical significance.

\section{Results}

Follow-up on rehospitalization. No patient was lost to follow-up during the subsequent 12 months. Fourteen respiratory-related events (10 IP acute exacerbations and 4 respiratory failures) occurred during the tracking period (event rate $=19.2 \%)$.

Participant characteristics. Table 1 shows clinical characteristics of participants in the two groups. There were no between-group significant differences in age, gender percentage, BMI, diagnosis, prednisolone use, forced expiratory volume $\left(\mathrm{FEV}_{1.0}\right), \Delta \mathrm{N}_{2}$, resting $\mathrm{SpO}_{2}, \Delta \mathrm{SpO}_{2}$, modified Borg scale at 6MWT end, and clinical laboratory data.

Correlation analysis. Lowest $\mathrm{SpO}_{2}, \Delta \mathrm{SpO}_{2}, \mathrm{SpO}_{2} 1$ min after 6MWT, distance, and $\mathrm{SpO}_{2}$ recovery index significantly correlated with \%DLco with moderate correlation coefficients. $\mathrm{SpO}_{2}$ recovery index obtained from 6 MWT had the highest correlation coefficient $(r=0.684, P<0.001$; Fig. 1$)$ including other evaluations.

ROC curves and related rehospitalization analysis. Figure 2 shows the ROC curve of $\mathrm{SpO}_{2}$ recovery index to deduce rehospitalization of respiratory-related events. AUC showing the usefulness of $\mathrm{SpO}_{2}$ recovery index usefulness was significantly higher at 0.75 (standard error $=0.07 ; P=0.005$ ). The optimum cutoff value 


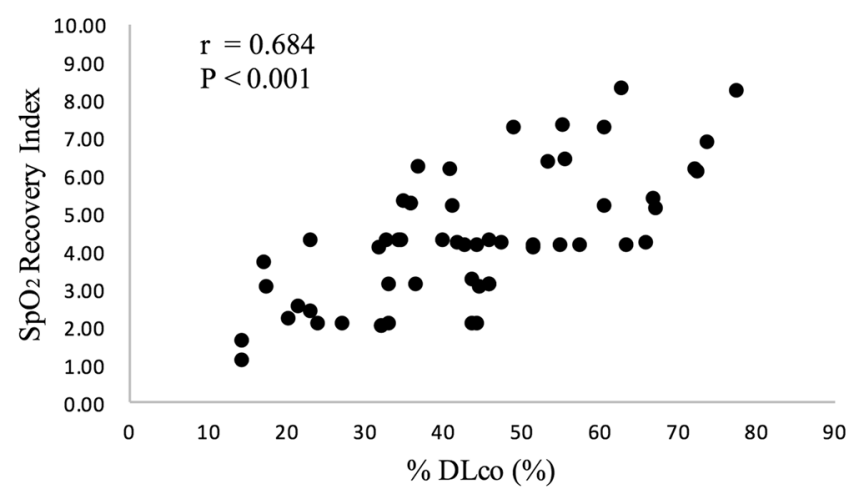

Figure 1. Correlations between \%DLco, $\mathrm{SpO}_{2}$ recovery index. \%DLco, DLco predicted; $\mathrm{SpO}_{2}$ recovery index, $\left\{\left(\mathrm{SpO}_{2} 1\right.\right.$ minute in to recovery after $6 \mathrm{MWT}$ minus lowest $\left.\mathrm{SpO}_{2}\right) /$ resting $\left.\mathrm{SpO}_{2}\right\} \times 100$.

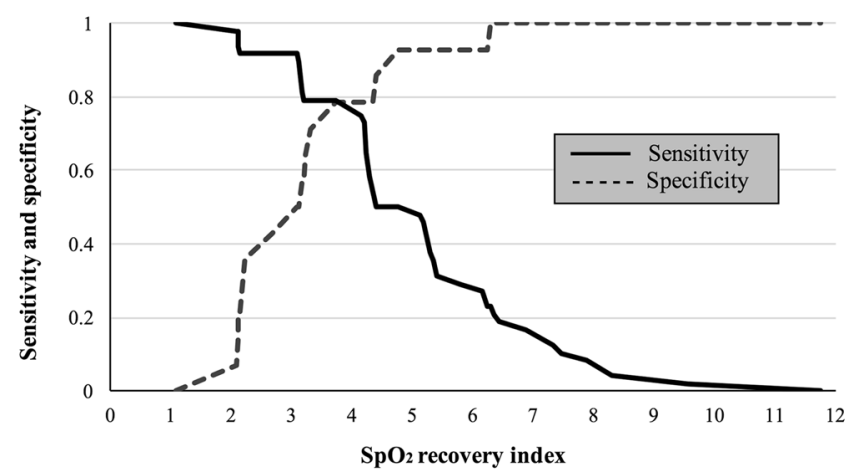

Figure 2. ROC curves to predict readmission of respiratory related events by $\mathrm{SpO}_{2}$ recovery index. Cutoff $4 \%$, AUC $0.75(\mathrm{P}=0.005)$, sensitivity $71.4 \%$, specificity $79.2 \%$, positive likelihood ratio 3.43 .

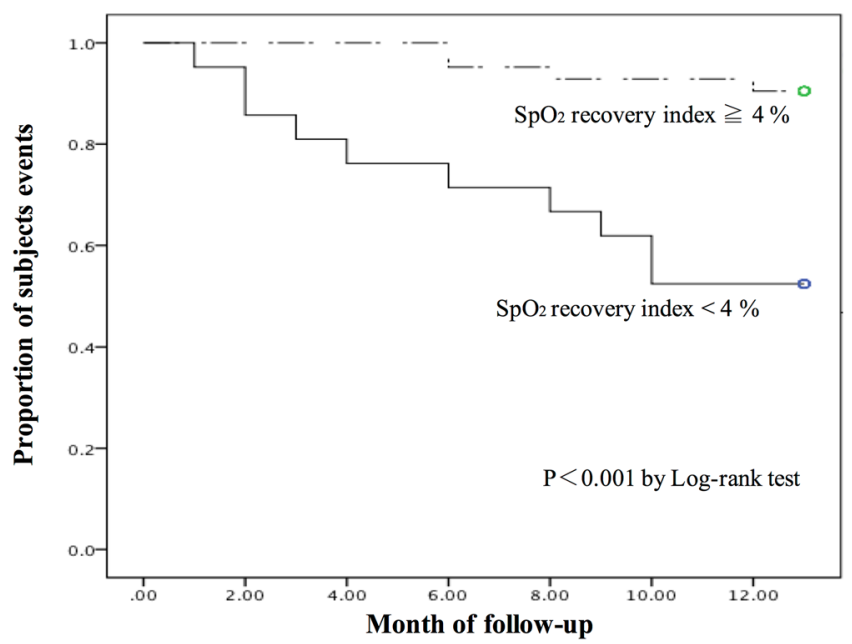

Figure 3. Cumulative event-free probability of patients with IP according to $\mathrm{SpO}_{2}$ recovery index estimated by the Kaplan-Meier method. Stratified according to whether $\mathrm{SpO}_{2}$ recovery index $\geqq 4 \%$ or $\mathrm{SpO}_{2}$ recovery index $<4 \%$.

of $\mathrm{SpO}_{2}$ recovery index was $4 \%$ (sensitivity $=71.4 \%$; specificity $=79.2 \%$ ). The positive likelihood ratio of in the cutoff of $\mathrm{SpO}_{2}$ recovery index was 3.43 .

Kaplan-Meier curves. Figure 3 shows Kaplan-Meier analyses of rehospitalization for the study sample stratified by $\mathrm{SpO}_{2}$ recovery index ( $\geq 4 \%$ vs $<4 \%$ ). In time-to-event survival analysis, $\mathrm{SpO}_{2}$ recovery index was significantly associated with respiratory-related events for patients with $\operatorname{IP}(P<0.001)$. 


\begin{tabular}{|c|c|c|c|c|c|c|c|c|}
\hline \multirow[b]{3}{*}{ Variable } & \multirow{2}{*}{\multicolumn{2}{|c|}{ Univariable analysis }} & \multicolumn{6}{|c|}{ Multivariate analysis } \\
\hline & & & \multicolumn{2}{|c|}{ Model 1} & \multicolumn{2}{|l|}{ Model 2} & \multicolumn{2}{|l|}{ Model 3} \\
\hline & HR $(95 \% \mathrm{CI})$ & P-value & HR (95\% CI) & P-value & HR $(95 \% \mathrm{CI})$ & $P$-value & HR $(95 \% \mathrm{CI})$ & $P$-value \\
\hline $\mathrm{SpO}_{2}$ recovery index $(\%)$ & $0.45(0.29-0.72)$ & 0.001 & $0.30(0.10-0.90)$ & 0.032 & - & - & - & - \\
\hline HRR1 (beat) & $0.84(0.90-0.97)$ & 0.032 & - & - & $0.91(0.82-0.99)$ & 0.045 & - & - \\
\hline 6MWT distance $(\mathrm{m})$ & $0.99(0.98-0.99)$ & 0.017 & - & - & - & - & \begin{tabular}{|l|}
1.00 \\
$(0.99-1.01)$
\end{tabular} & 0.810 \\
\hline \multicolumn{9}{|l|}{ Demographics } \\
\hline Age (years) & $0.97(0.91-1.03)$ & 0.307 & ND & ND & ND & ND & ND & ND \\
\hline Sex (men vs women) & $1.01(0.34-3.34)$ & 0.980 & ND & ND & ND & ND & ND & ND \\
\hline $\operatorname{BMI}\left(\mathrm{kg} / \mathrm{m}^{2}\right)$ & $0.91(0.75-1.10)$ & 0.358 & ND & ND & ND & ND & ND & ND \\
\hline Diagnosis & $0.91(0.44-1.91)$ & 0.812 & ND & ND & ND & ND & ND & ND \\
\hline $\mathrm{PH}$ (yes versus no) & $1.41(0.47-4.20)$ & 0.538 & ND & ND & ND & ND & ND & ND \\
\hline \multicolumn{9}{|l|}{ Treatment } \\
\hline Use PSL (mg/day) & $1.00(0.97-1.03)$ & 0.920 & ND & ND & ND & ND & ND & ND \\
\hline $\mathrm{O}_{2}$ flow $(\mathrm{L} / \mathrm{min})$ & $1.69(1.01-2.62)$ & 0.018 & ND & ND & ND & ND & ND & ND \\
\hline \multicolumn{9}{|l|}{ Physiologic } \\
\hline FVC predicted (\%) & $0.94(0.89-0.98)$ & 0.003 & ND & ND & ND & ND & ND & ND \\
\hline $\mathrm{FEV}_{1.0}(\%)$ & $0.96(0.93-1.00)$ & 0.065 & ND & ND & ND & ND & ND & ND \\
\hline DLco predicted (\%) & $0.94(0.90-0.98)$ & 0.005 & ND & ND & ND & ND & ND & ND \\
\hline$\Delta \mathrm{N}_{2}(\%)$ & $1.20(0.93-1.56)$ & 0.163 & ND & ND & ND & ND & ND & ND \\
\hline Resting $\mathrm{SpO}_{2}(\%)$ & $0.77(0.55-1.06)$ & 0.111 & ND & ND & ND & ND & ND & ND \\
\hline Lowest $\mathrm{SpO}_{2}(\%)$ & $0.93(0.84-1.02)$ & 0.126 & ND & ND & ND & ND & ND & ND \\
\hline$\Delta \mathrm{SpO}_{2}(\%)$ & $1.06(0.96-1.16)$ & 0.270 & ND & ND & ND & ND & ND & ND \\
\hline $\mathrm{SpO}_{2} 1$ minute (\%) & $0.91(0.84-0.99)$ & 0.029 & ND & ND & ND & ND & ND & ND \\
\hline Modifide Borg Scale & $1.21(0.98-2.18)$ & 0.067 & ND & ND & ND & ND & ND & ND \\
\hline \multicolumn{9}{|l|}{ Laboratory data } \\
\hline KL-6 (U/ml) & $1.00(1.00-1.01)$ & 0.365 & ND & ND & ND & ND & ND & ND \\
\hline $\mathrm{P} / \mathrm{F}$ ratio & $0.99(0.98-1.00)$ & 0.073 & ND & ND & ND & ND & ND & ND \\
\hline Propensity score & ND & ND & $2.90(0.25-33.2)$ & 0.392 & $1.13(0.11-11.9)$ & 0.92 & \begin{tabular}{|l|}
0.09 \\
$(0.09-1.01)$
\end{tabular} & 0.51 \\
\hline
\end{tabular}

Table 2. Cox Proportional Hazard Respiratory Related Events Analysis. Multivariable analysis indicates the adjusted effect by applying propensity score which is a conditional probability given by other clinicopathologic factors including age, sex, BMI, diagnosis, $\mathrm{PH}$, use PSL, Supplemental $\mathrm{O}_{2}$ flow, FVC predicted, $\mathrm{FEV}_{1.0}$, DLco predicted, $\Delta \mathrm{N}_{2}$, Resting $\mathrm{SpO}_{2}$, Lowest $\mathrm{SpO}_{2}, \Delta \mathrm{SpO}_{2}, \mathrm{SpO}_{2} 1$ minute, Modifide Borg Scale, KL-6, P/F ratio. Definition of abbreviations: $\mathrm{HR}$, hazard ratio; $\mathrm{CI}$, indicates confidence interval; $\mathrm{ND}$, not done; $\mathrm{SpO}_{2}$ recovery index; $\left\{\left(\mathrm{SpO}_{2} 1\right.\right.$ minute in to recovery after 6MWT minus lowest $\left.\mathrm{SpO}_{2}\right) /$ resting $\left.\mathrm{SpO}_{2}\right\} \times 100 ; \mathrm{HRR} 1, \mathrm{HR}$ at the end of 6MWT minus HR after 1 minute at the end of 6MWT; BMI, body mass index; PH, Pulmonary hypertension; PSL, Prednisolone; $\mathrm{O}_{2}$ flow, Supplemental $\mathrm{O}_{2}$ flow; $\Delta \mathrm{SpO}_{2}, \mathrm{SpO}_{2}$ at the end of the $6 \mathrm{MWT}$ minus $\mathrm{SpO}_{2}$ at baseline; $\mathrm{SpO}_{2} 1$ minute, $\mathrm{SpO}_{2} 1$ min into recovery after 6MWT; KL-6, Krebs von den Lungen-6; P/F ratio, $\mathrm{PaO}_{2} / \mathrm{FIO}_{2}$ ratio.

Multivariate Cox regression analysis. Table 2 shows a Cox proportional hazard model. In multivariable analyses using the propensity score, $\mathrm{SpO}_{2}$ recovery index [hazard ratio $(\mathrm{HzR})=0.3 ; 95 \%$ confidence inter$\operatorname{val}(\mathrm{CI})=0.10-0.90 ; P=0.032]$ and $\mathrm{HHR} 1(\mathrm{HzR}=0.91 ; 95 \% \mathrm{CI}=0.82-0.99 ; P=0.045)$ were significantly associated with respiratory-related events. Among them, $\mathrm{SpO}_{2}$ recovery index was the most associated with respiratory-related events.

\section{Discussion}

To the best of our knowledge, this is the first study to reveal a relationship between $\mathrm{SpO}_{2}$ recovery index values obtained from 6MWT and pulmonary diffusion capacities in patients with IP. It is also the first exploration of the predictors of rehospitalization predictors due to acute IP exacerbation and respiratory failure. $\mathrm{SpO}_{2}$ recovery index from 6MWT for IP inpatients was most strongly associated with pulmonary diffusion capacity and was a strong predictor for rehospitalization due to acute IP exacerbation and respiratory failure. When the $4 \%$ cutoff generated from ROC curves was used as a reference for $\mathrm{SpO}_{2}$ recovery index value for rehospitalization, the risk of rehospitalization during the follow-up period decreased by 0.3 -fold with every $1 \%$ increase in $\mathrm{SpO}_{2}$ recovery index. Multivariate Cox regression analysis identified HRR1 obtained from 6MWT as a rehospitalization predictor for respiratory-related events, supporting previous studies on mortality. However, in this study, $\mathrm{SpO}_{2}$ recovery index was as a stronger predictor than HRR1.

Patients with IP with EIH have a poor prognosis, indicating the importance of evaluating $\mathrm{EIH}^{5,27}$. In clinical, radiological and physiological scoring systems for predicting prognosis in patients with IP, $\mathrm{PaO}_{2}$ during cardiopulmonary exercise testing is a significant survival rate predictor and the strongest prognosis predictor ${ }^{5}$. A recent 
American Thoracic Society consensus statement suggested a 4\% decrease in saturation during exercise as an adverse prognostic sign in idiopathic pulmonary fibrosis (IPF) ${ }^{1}$. Many of these studies focused on desaturation/ $\mathrm{PaO}_{2}$ during cardiopulmonary exercise testing. However, registry data suggest that cardiopulmonary exercise tests are rarely used to assess prognostic predictions in IPF patients ${ }^{28}$, possibly relating to the expense and limited availability of this diagnostic modality. $6 \mathrm{MWT}$ is a simple, convenient, inexpensive test requiring minimal medical personnel and can be performed in an office setting ${ }^{29}$. In this study, clinical data derived from $6 \mathrm{MWT}$ were analyzed. $\mathrm{SpO}_{2}$ recovery index from $6 \mathrm{MWT}$ was significantly associated with the gas exchange index \%DLco, suggesting the estimation of pulmonary diffusion capacity levels using $\mathrm{SpO}_{2}$ recovery index after $6 \mathrm{MWT}$ without performing detailed pulmonary function tests (difficult to conduct due to insufficient facilities and environmental problems). These results may help in risk management during exercise therapy and determining oxygen levels during home oxygen therapy introduction.

For rehospitalization predictors, causes of deaths in patients with IP were not specifically identified in previous studies. HR changes during exercise and HRR after exercise, demonstrated in many previous studies, may reflect cardiac function and are presumed to be outcomes, a large part of which is accounted for by mortality attributable to cardiac disease as a complex. Previous studies included large patient numbers with high right ventricular systolic pressure (RSVP), using cardiac medication ${ }^{14}$, and who suffered $\mathrm{PH}$ after the study/had $\mathrm{PH}$-related mortality ${ }^{13}$. To explain HR changes during and HRR changes after exercise as mortality predictors, Heindl ${ }^{30}$ noted abnormal sympathetic activation in patients with chronic respiratory failure, including some with pulmonary fibrosis. Most deaths occur from the progression of lung fibrosis rather than from commonly occurring comorbidities $^{15-17}$. Frequent hospitalization for respiratory problems are common and associated with death ${ }^{15-17}$. Identifying rehospitalization predictors due to respiratory-related events (respiratory failure and acute IP exacerbation) was necessary. Unlike previous studies, our study limited the reasons for rehospitalization to IP exacerbation and respiratory-related events, including respiratory failure. Respiratory system function had a greater impact on the analysis and may justify why $\mathrm{SpO}_{2}$ recovery index after $6 \mathrm{MWT}$, which is more relevant to the pulmonary diffusion capacity than HHR1, was a rehospitalization predictor for respiratory-related events. $\mathrm{SpO}_{2}$ recovery index was a useful index with clinical applicability regardless of whether the resting $\mathrm{SpO}_{2}$ level was high/ low because it was adjusted with resting $\mathrm{SpO}_{2}$ for compensating oxygen consumption effect.

These results allow for a novel means of predicting respiratory-related events, such as acute IP exacerbation and respiratory failure and all-cause mortality, including that due to cardiac diseases. Evaluating $\mathrm{SpO}_{2}$ recovery index after $6 \mathrm{MWT}$ predicts rehospitalization due to acute IP exacerbation and respiratory failure, aiding in clinical practice.

Limitations of this study are that independent variables used in the multivariate analysis were limited because of the small sample size and the short, 1-year follow-up period, which can be addressed by subject accumulation and longer follow-up. This study excluded patients with immeasurable 6MWT, such as those using a high-flow-rate oxygenator and with a history of bone and joint diseases. Therefore, this method is not applicable to all patients in clinical practice and should be used for prognosis prediction after appropriate patient selection. $\mathrm{SpO} 2$ recovery is useful in predicting rehospitalization due to respiratory-related events. However, there is no clear intervention for minimizing rehospitalization due to respiratory-related events risk, and improvement of exercise tolerance and lower leg muscle strength by rehabilitation may be important, other than pulmonary diffusion capacity due to drugs.

\section{Conclusion}

$\mathrm{SpO}_{2}$ recovery index after $6 \mathrm{MWT}$ in hospitalized patients with IP was most closely related to pulmonary diffusion capacity and the strongest independent rehospitalization predictor due to respiratory-related events. In clinical practice, pulmonary diffusion capacity can be estimated using $\mathrm{SpO}_{2}$ recovery indices obtained from 6MWT in appropriately selected patients and may help during exercise therapy. It was also useful in predicting rehospitalization due to respiratory-related events.

Received: 12 December 2018; Accepted: 8 October 2019;

Published online: 23 October 2019

\section{References}

1. American Thoracic Society. idiopathic pulmonary fibrosis: diagnosis and treatment: international consensus statement: American Thoracic Society (ATS), and the European Respiratory Society (ERS). Am J Respir Crit Care Med. 161, 646-664 (2006).

2. Swigris, J. J., Kuschner, W. G., Jacobs, S. S., Wilson, S. R. \& Gould, M. K. Health-related quality of life in patients with idiopathic pulmonary fibrosis: a systematic review. Thorax. 60, 588-594 (2005).

3. Hamada, K. et al. Significance of pulmonary arterial pressure and diffusion capacity of lung as prognosticator in patients with idiopathic pulmonary fibrosis. Chest. 131, 650-656 (2007).

4. Flaherty, K. R. et al. Ideopathic pulmonary fibrosis. Prognostic value of changes in physiology and six minute-walk test. Am J Repir Crit Care Med. 174, 803-809 (2006).

5. King, T. E. Jr., Tooze, J. A., Schwarz, M. I., Brown, K. R. \& Cherniack, R. M. Predicting survival in idiopathic pulmonary fibrosis: scoring system and survival model. Am J Respir Crit Care Med. 164, 1171-1181 (2001).

6. Lama, V. N. et al. Prognostic Value of Desaturation during a 6-Minute Walk Test in Idiopathic Interstitial Pneumonia. Am J Respir Crit Care Med. 168, 1084-1090 (2003).

7. Lamberto, C. et al. Membrane and capillary blood components of diffusion capacity of the lung for carbon monoxide in pulmonary sarcoidosis. Chest. 125, 2061-2068 (2004).

8. Lebecque, P., Lapierre, J. G., Lamarre, A. \& Coates, A. L. Diffusion capacity and oxgen desaturation effects on exercise in patients with cystic fibrosis. Chest. 91, 693-697 (1987).

9. Solway, S., Brooks, D., Lacasse, Y. \& Thomas, S. A qualitative systematic overview of the measurement properties of functional walk tests used in the cardiorespiratory domain. Chest. 119, 256-270 (2001).

10. Steele, B. Timed walk tests of exercise capacity in chronic cardiopulmonary illness. J Cardiopulm Rehabil. 16, 25-33 (1996). 
11. Cahalin, L. P. et al. Heart rate recovery after the $6 \mathrm{~min}$ walk test rather than distance ambulated is a powerfulprognostic indicator in heart failure with reduced and preserved ejection fraction: a comparison with cardiopulmonary exercise testing. European Journal of Heart Failure. 15, 519-527 (2013).

12. Lacasse, M. et al. Post-exercise heart rate recovery and mortality in chronic obstructive pulmonary disease. Respir Med. 99, 877-886 (2005).

13. Swigris, J. J. et al. Heart rate recovery after six-minute walk test predicts pulmonary hypertension in patients with idiopathic pulmonary fibrosis. Respirology. 16, 439-445 (2011).

14. Swigris, J. J. et al. Heart Rate Recovery After 6-Min Walk Test Predicts Survival in Patients With Idiopathic Pulmonary Fibrosis. Chest. 136, 841-848 (2009).

15. Martinez, F. J. et al. The clinical course of patients with idiopathic pulmonary fibrosis. Ann Intern Med. 142, 963-967 (2005).

16. King, T. E. Jr. et al. Effect of interferon gamma-1b on survival in patients with idiopathic pulmonary fibrosis (INSPIRE): a multicentre, randomised, placebo-controlled trial. Lancet. 374, 222-228 (2009).

17. Brown, A. et al. Outcomes after hospitalization in idiopathic pulmonary fibrosis: a cohort study. Chest. 147, 173-179 (2015).

18. American Thoracic Society/European Respiratory Society. American Thoracic Society/European Respiratory Society International Multidisciplinary Consensus Classification of the Idiopathic Interstitial Pneumonias. Am J Respir Crit Care Med. 165, 277-304 (2002).

19. Avin, K. G. et al. Management of falls in community-dwelling older adults: clinical guidance statement from the Academy of Geriatric Physical Therapy of the American Physical Therapy Association. Physical therapy. 95, 815-834 (2015).

20. Gay, S. E. et al. Idiopathic pulmonary fibrosis: predicting response to therapy and survival. Am J Respir Crit Care Med. 157, 1063-1072 (1998).

21. Macintyre, N. et al. Standardisation of the single-breath determination of carbon monoxide uptake in the lung. Eur Respir J. 26, 720-735 (2005)

22. Miller, M. R. et al. Standardisation of spirometry. Eur Respir J. 26, 319-338 (2005).

23. Pellegrino, R. et al. Interpretative strategies for lung function tests. Eur Respir J. 26, 948-968 (2005).

24. Guidelines for Treatment of Pulmonary Hypertension. Japan Circulation Society. 1-69 (2012).

25. ATS Statement: Guidelines for the Six-Minute Walk Test. Am J Repir Crit Care Med. 166, 111-117 (2002).

26. Hosmer, D. \& Lemeshow, S. Applied logistic regression. New York: John Wiley and Sons (2000).

27. Miki, K. et al. Impairments and prognostic factors for survival in patients with idiopathic pulmonary fibrosis. Respir Med. 97, 482-490 (2003).

28. Mapel, D. W., Samet, J. M. \& Coultas, D. B. Corticosteroids and the treatment of idiopathic pulmonary fibrosis: past, present, and future. Chest. 110, 1058-1067 (1996).

29. Poulain, M. et al. 6-Minute walk testing is more sensitive than maximal incremental cycle testing for detecting oxygen desaturation in patients with COPD. Chest. 123, 1401-1407 (2003).

30. Heindl, S. et al. Marked sympathetic activation in patients with chronic respiratory failure. Am J Respir Crit Care Med. 164, 597-601 (2001).

\section{Acknowledgements}

The authors would like to thank Enago for the English language review.

\section{Author contributions}

Yasunari sakai designed the study, and wrote the initial draft of the manuscript. Yasunari sakai contributed to analysis and interpretation of data, and assisted in the preparation of the manuscript. Mr. Yamamoto, Mrs. Hoshina, and Mr. Kawachi contributed to the data collection. Mr. Yamamoto contributed to the statistical analysis. Mr. Yamamoto, Mrs. Hoshina, Mr. Kawachi, Mr. Ichiyama and Mr. Hanaoka have contributed to data collection and interpretation, and critically reviewed the manuscript. All authors approved the final version of the manuscript, and agree to be accountable for all aspects of the work in ensuring that questions related to the accuracy or integrity of any part of the work are appropriately investigated and resolved.

\section{Competing interests}

The authors declare no competing interests.

\section{Additional information}

Correspondence and requests for materials should be addressed to Y.S.

Reprints and permissions information is available at www.nature.com/reprints.

Publisher's note Springer Nature remains neutral with regard to jurisdictional claims in published maps and institutional affiliations.

(c) (i) Open Access This article is licensed under a Creative Commons Attribution 4.0 International

License, which permits use, sharing, adaptation, distribution and reproduction in any medium or format, as long as you give appropriate credit to the original author(s) and the source, provide a link to the Creative Commons license, and indicate if changes were made. The images or other third party material in this article are included in the article's Creative Commons license, unless indicated otherwise in a credit line to the material. If material is not included in the article's Creative Commons license and your intended use is not permitted by statutory regulation or exceeds the permitted use, you will need to obtain permission directly from the copyright holder. To view a copy of this license, visit http://creativecommons.org/licenses/by/4.0/.

(C) The Author(s) 2019 